\title{
Hydrodynamic fluctuations in the Kolmogorov flow: Linear regime
}

\author{
I. Bena ${ }^{a}$, M. Malek Mansour ${ }^{b}$ and F. Baras ${ }^{b}$ \\ (a) Limburgs Universitair Centrum \\ B-3590 Diepenbeek, Belgium \\ (b) Centre for Nonlinear Phenomena and Complex Systems \\ Université Libre de Bruxelles, Campus Plaine, C.P. 231 \\ B-1050 Brussels, Belgium
}

(October 28, 2018)

\begin{abstract}
The Landau-Lifshitz fluctuating hydrodynamics is used to study the statistical properties of the linearized Kolmogorov flow. The relative simplicity of this flow allows a detailed analysis of the fluctuation spectrum from near equilibrium regime up to the vicinity of the first convective instability threshold. It is shown that in the long time limit the flow behaves as an incompressible fluid, regardless of the value of the Reynolds number. This is not the case for the short time behavior where the incompressibility assumption leads in general to a wrong form of the static correlation functions, except near the instability threshold. The theoretical predictions are confirmed by numerical simulations of the full nonlinear fluctuating hydrodynamic equations.
\end{abstract}

05.40+j, 05.90+m, 47.20.-k, 42.70.Ft, 47.40.Dc 


\section{INTRODUCTION}

A common theoretical approach for the study of fluctuations is the Landau-Lifshitz fluctuating hydrodynamics [1] mainly because of its relative simplicity as compared to more fundamental approaches [2,3]. Fluctuating hydrodynamics is a stochastic formulation of standard fluid mechanics. Spontaneous fluctuations of hydrodynamic variables are introduced into the transport equations by adding random components to the dissipative part of the pressure and heat fluxes. Since these fluxes are not conserved quantities, the correlations of the random terms are expected to be short-ranged and short-lived, so that on a hydrodynamic scale they are assumed to be Dirac-delta correlated. Their strengths are then chosen to yield the correct equilibrium thermodynamic fluctuations as derived from the Gibbs distribution.

Fluctuating hydrodynamics has been used by various authors to study the statistical properties of simple fluids subjected to nonequilibrium constraints, such as temperature gradient [2, [4,5] or shear [3,6] (for a review, see ref. [7]). Recent light scattering results, obtained for systems under temperature gradient, have shown quantitative agreement with theoretical predictions [8]. Quantitative agreements have also been demonstrated with results based on particle simulations, both for systems under temperature gradient [9,10] and shear [1]. A more important issue is obviously the role of fluctuations in the onset of hydrodynamical instabilities, such as the convective instability arising in the Bénard problem [12].

The macroscopic studies of sub-sonic hydrodynamical instabilities are usually based on the incompressibility assumption. However, as first pointed out by Zaitsev and Shliomis [13], this assumption is basically inconsistent with the very foundation of the fluctuating hydrodynamics formalism since it imposes fictitious correlations between the velocity components of the fluids. On the other hand, even in the absence of noise, the mathematical analysis of convective instabilities arising in compressible fluids proves to be quite involved [14,15. One way to overcome this difficulty is to look for idealized models which, in spite 
of their extreme simplicity, can nevertheless lead to hydrodynamical instabilities analog to those observed in real systems. Our main purpose in this article is to study the statistical properties of one such a model proposed some fifty years ago by Kolmogorov [16]. As we will show, the periodic boundary conditions associated to this model allow detailed analysis of the fluctuation spectrum from near equilibrium up to the vicinity of the first instability leading to convective rolls.

The Kolmogorov flow will be presented in the next section where some well known aspects of its macroscopic behavior are reviewed. The statistical properties of the model will be discussed in section III. We will show that the dynamic structure factor of the fluid is practically not affected by nonequilibrium constraints. This is not the case for the velocity correlation functions which become long-ranged as soon as the system is driven out of equilibrium. Their amplitude is shown to diverges as one approaches to the convective instability threshold. Conclusions and perspectives will be presented in section IV.

\section{KOLMOGOROV FLOW}

Consider an isothermal flow in a rectangular box $L_{x} \times L_{y}$ oriented along the main axes, that is $\left\{0 \leq x<L_{x}, 0 \leq y<L_{y}\right\}$. Periodic boundary conditions are assumed in both directions and the flow is maintained through an external force field of the form

$$
\mathbf{F}_{\text {ext }}=F_{0} \sin \left(2 \pi n y / L_{y}\right) \mathbf{1}_{x}
$$

where $\mathbf{1}_{x}$ is the unit vector in the $x$ direction. This model represents the so-called "Kolmogorov flow" and it belongs to the wider class of two-dimensional negative effective eddy viscosity flows [17]. It is entirely characterized through the strength of the force field $F_{0}$, the parameter $n$, which controls the wave number of the forcing, and the aspect ratio $a_{r}$, defined as

$$
a_{r}=L_{x} / L_{y}
$$

In the following, we will mainly concentrate on the case $n=1$. 
The hydrodynamic equations for this model read:

$$
\begin{gathered}
\frac{\partial \rho}{\partial t}=-\boldsymbol{\nabla} \cdot(\rho \mathbf{v}) \\
\rho \frac{\partial \mathbf{v}}{\partial t}=-\rho(\mathbf{v} \cdot \boldsymbol{\nabla}) \mathbf{v}-\boldsymbol{\nabla} P-\boldsymbol{\nabla} \cdot \boldsymbol{\sigma}+\mathbf{F}_{e x t}
\end{gathered}
$$

where $\rho$ is the mass density, $P$ the hydrostatic pressure and $\boldsymbol{\sigma}$ the two dimensional stress tensor:

$$
\sigma_{i, j}=-\eta\left(\frac{\partial v_{i}}{\partial x_{j}}+\frac{\partial v_{j}}{\partial x_{i}}-\delta_{i, j} \boldsymbol{\nabla} \cdot \mathbf{v}\right)-\zeta \delta_{i, j} \boldsymbol{\nabla} \cdot \mathbf{v}
$$

For simplicity, we shall assume that the shear and bulk viscosity coefficients, $\eta$ and $\zeta$, are state independent, i.e. they are constant. It can then be easily checked that at the stationary state the pressure and the density are uniform in space $\left(P_{s t}=P_{0}, \rho_{s t}=\rho_{0}\right)$ whereas the velocity profile is given by:

$$
\begin{gathered}
\mathbf{v}_{s t}=u_{0} \sin \left(2 \pi y / L_{y}\right) \mathbf{1}_{x} \\
u_{0}=\frac{F_{0} L_{y}^{2}}{4 \pi^{2} \eta}
\end{gathered}
$$

For small enough $F_{0}$, this stationary flow is stable. As we increase $F_{0}$, however, the flow may become unstable giving rise to rotating convective patterns. Other instabilities of increasing complexity may occur for larger values of $F_{0}$, culminating in a turbulent - like behavior [18 20]. In this paper we shall limit ourselves to the analysis of the system before its first instability.

We still have to supply the momentum conservation equation, eq.(四), with an equation of state relating the pressure to the density (recall that the system is isothermal). In this section, we follow the traditional macroscopic analysis by assuming that the flow is incompressible, i.e.

$$
\boldsymbol{\nabla} \cdot \mathbf{v}=\frac{\partial u}{\partial x}+\frac{\partial v}{\partial y}=0
$$

where $u$ and $v$ represent the $x$ and $y$ components of the velocity, respectively, i.e. $\mathbf{v} \equiv$ $u \mathbf{1}_{x}+v \mathbf{1}_{y}$. Relation (7) implies a uniform density $\rho_{0}$ throughout the system for all times, if 
initially so, as well as the existence of a scalar function $\psi(x, y)$, known as "stream function", defined by the relations:

$$
u=\frac{\partial \psi}{\partial y}, \quad v=-\frac{\partial \psi}{\partial x}
$$

Scaling lengths by $L_{y}$, velocities by $u_{0}$ and time by $L_{y} / u_{0}$, the dimensionless equation for the stream function reads:

$$
\frac{\partial\left(\nabla^{2} \psi\right)}{\partial t}=-\frac{\partial \psi}{\partial y} \frac{\partial\left(\nabla^{2} \psi\right)}{\partial x}+\frac{\partial \psi}{\partial x} \frac{\partial\left(\nabla^{2} \psi\right)}{\partial y}+R^{-1} \nabla^{2}\left(\nabla^{2} \psi\right)+8 \pi^{3} R^{-1} \cos (2 \pi y)
$$

where $R$ represents the Reynolds number,

$$
R=\frac{u_{0} L_{y}}{\nu}
$$

and $\nu \equiv \eta / \rho$ is the kinematic viscosity. The stationary solution of (9) reads:

$$
\psi_{s t}=-\frac{1}{2 \pi} \cos (2 \pi y)
$$

Setting $\psi=\psi_{s t}+\delta \psi$, and linearizing (9) around $\psi_{s t}$, one gets

$$
\frac{\partial\left(\nabla^{2} \delta \psi\right)}{\partial t}=-\sin (2 \pi y) \frac{\partial\left(\nabla^{2} \delta \psi\right)}{\partial x}-4 \pi^{2} \sin (2 \pi y) \frac{\partial \delta \psi}{\partial x}+R^{-1} \nabla^{2}\left(\nabla^{2} \delta \psi\right)
$$

Owing to periodic boundary conditions, $\delta \psi(x, y, t)$ can be expanded in Fourier series:

$$
\begin{aligned}
\delta \psi(x, y, t) & =\sum_{k_{x}, k_{y}=-\infty}^{\infty} \exp \left(-2 p i i k_{y} y\right) \exp \left(-2 \pi i k_{x} x / a_{r}\right) \delta \psi_{k_{x}, k_{y}}(t), \\
\delta \psi_{k_{x}, k_{y}}(t) & =\int_{0}^{1} d y \exp \left(2 \pi i k_{y} y\right) \frac{1}{a_{r}} \int_{0}^{a_{r}} d x \exp \left(2 \pi i k_{x} x / a_{r}\right) \delta \psi(x, y, t)
\end{aligned}
$$

Equation (12) can then be transformed to

$$
\begin{aligned}
\frac{\partial \delta \psi_{k_{x}, k_{y}}}{\partial t}= & -4 \pi^{2} R^{-1}\left(\tilde{k}_{x}^{2}+k_{y}{ }^{2}\right) \delta \psi_{k_{x}, k_{y}} \\
& +\pi \tilde{k}_{x}\left[\delta \psi_{k_{x}, k_{y}+1}-\delta \psi_{k_{x}, k_{y}-1}\right] \\
& +2 \pi \frac{\tilde{k}_{x} k_{y}}{\tilde{k}_{x}^{2}+k_{y}{ }^{2}}\left[\delta \psi_{k_{x}, k_{y}+1}+\delta \psi_{k_{x}, k_{y}-1}\right]
\end{aligned}
$$


where we have set

$$
\tilde{k}_{x}=k_{x} / a_{r}
$$

In its general form, the analysis of this equation proves to be quite difficult [21]. On the other hand, if $\psi_{s t}$ is stable then, in the long time limit, the evolution of the system will be mainly governed by long wave length modes. Accordingly, we start our analysis by considering only the modes $k_{y}=0, \pm 1$, i.e. we assume that $\delta \psi_{k_{x}, k_{y}}(t) \approx 0$ for $\left|k_{y}\right| \geq 2[22$. The equation (14) then reduces to a $3 \times 3$ matricial equation whose eigenvalues for $k_{x} \neq 0$ (the case $k_{x}=0$ is trivial) are found to be :

$$
\begin{aligned}
& \lambda_{1}=-2 \pi^{2} R^{-1}\left(1+2 \tilde{k}_{x}^{2}\right)+\pi \sqrt{2 \tilde{k}_{x}^{2}\left(1-\tilde{k}_{x}^{2}\right) /\left(1+\tilde{k}_{x}^{2}\right)+4 \pi^{2} R^{-2}}, \\
& \lambda_{2}=-2 \pi^{2} R^{-1}\left(1+2 \tilde{k}_{x}^{2}\right)-\pi \sqrt{2 \tilde{k}_{x}^{2}\left(1-\tilde{k}_{x}^{2}\right) /\left(1+\tilde{k}_{x}^{2}\right)+4 \pi^{2} R^{-2}}, \\
& \lambda_{3}=-4 \pi^{2} R^{-1}\left(1+\tilde{k}_{x}^{2}\right) .
\end{aligned}
$$

It follows from (16) that $\lambda_{2}$ and $\lambda_{3}$ are always negative, whereas there exists a critical value of the Reynolds number

$$
R_{c}\left(k_{x}\right)=2 \sqrt{2} \pi \frac{1+\tilde{k}_{x}^{2}}{\sqrt{1-\tilde{k}_{x}^{2}}} ; \quad 0<\tilde{k}_{x}^{2} \leq 1
$$

for which $\lambda_{1}$ becomes equal to zero, thus indicating the limit of the stability of the corresponding mode [23]. Clearly $R_{c}$ is an increasing function of $\left|k_{x}\right|$ so that the first modes to become unstable correspond to $\left|k_{x}\right|=1$, provided the aspect ratio $a_{r}>1$. As $a_{r} \rightarrow 1$, $R_{c} \rightarrow \infty$, indicating that no instability can develop for perturbations of the same spatial periodicity as the applied force (see ref. [24]). In the following, we shall therefore concentrate on the case $a_{r}>1$.

For $a_{r}=2$, relation (17) predicts a critical Reynolds number $R_{c}=5 \pi \sqrt{6} / 3 \approx 12.8255$. Analytical calculations can still be handled when the modes $k_{y}= \pm 2, \pm 3$ are taken into account as well, and lead to a critical Reynolds number $R_{c} \approx 12.8736$. The discrepancy thus remains smaller than $0.4 \%$. Numerical evaluation of $R_{c}$ performed with a total amount of 103 modes shows no further discrepancy. We thus conclude that one can rely reasonably 
well on a "3-modes" approximation theory (that is $\delta \psi_{k_{x}, k_{y}}(t) \approx 0$ for $\left|k_{y}\right| \geq 2$ ), as long as $R$ is close to $R_{c}$. We shall use this approximation in the next section to study the statistical properties of the system near its first instability.

\section{HYDRODYNAMIC FLUCTUATIONS}

To study the fluctuations spectrum, we first linearize the hydrodynamic equations (3), (1) around the stationary state. Setting $\rho=\rho_{0}+\delta \rho, P=P_{0}+\delta P$ and $\mathbf{v}=\mathbf{v}_{s t}+\delta \mathbf{v}$, and following Landau and Lifshitz [1], the fluctuating hydrodynamic equations read:

$$
\begin{aligned}
\frac{\partial \delta \rho}{\partial t} & =-\rho_{0}\left(\frac{\partial \delta u}{\partial x}+\frac{\partial \delta v}{\partial y}\right)-u_{0} \sin \left(2 \pi y / L_{y}\right) \frac{\partial \delta \rho}{\partial x} \\
\rho_{0} \frac{\partial \delta \mathbf{v}}{\partial t} & =-\rho_{0}\left(\mathbf{v}_{s t} \cdot \boldsymbol{\nabla}\right) \delta \mathbf{v}-\rho_{0}(\delta \mathbf{v} \cdot \boldsymbol{\nabla}) \mathbf{v}_{s t}-\boldsymbol{\nabla} \delta P-\boldsymbol{\nabla} \cdot \delta \boldsymbol{\sigma}
\end{aligned}
$$

$\delta \boldsymbol{\sigma}$ is the two dimensional fluctuating stress tensor:

$$
\delta \sigma_{i, j}=-\eta\left(\frac{\partial \delta v_{i}}{\partial x_{j}}+\frac{\partial \delta v_{j}}{\partial x_{i}}-\delta_{i, j} \boldsymbol{\nabla} \cdot \delta \mathbf{v}\right)-\zeta \delta_{i, j} \boldsymbol{\nabla} \cdot \delta \mathbf{v}+S_{i, j}
$$

where $\mathbf{S}$ is a random tensor whose elements $\left\{S_{i, j}\right\}$ are Gaussian white noises with zero mean and covariances given by

$$
<S_{i, j}(\mathbf{r}, t) S_{k, \ell}\left(\mathbf{r}^{\prime}, t^{\prime}\right)>=2 k_{B} T_{0} \delta\left(t-t^{\prime}\right) \delta\left(\mathbf{r}-\mathbf{r}^{\prime}\right)\left[\eta\left(\delta_{i, k}^{K r} \delta_{j, \ell}^{K r}+\delta_{i, \ell}^{K r} \delta_{j, k}^{K r}\right)+(\zeta-\eta) \delta_{i, j}^{K r} \delta_{k, \ell}^{K r}\right]
$$

We still have to specify the equation of state. Since the fluid is isothermal, we simply set

$$
\delta P=c_{s}^{2} \delta \rho
$$

where $c_{s}$ is the isothermal sound speed. Scaling lengths by $L_{y}$, time by $L_{y} / c_{s}, \delta \rho$ by $\rho_{0}$ and $\delta \mathbf{v}$ by the speed of sound, the dimensionless fluctuating equations in the Fourier space read:

$$
\begin{aligned}
\frac{\partial \delta \rho_{k_{x}, k_{y}}(t)}{\partial t}=2 \pi & i\left(\tilde{k}_{x} \delta u_{k_{x}, k_{y}}+k_{y} \delta v_{k_{x}, k_{y}}\right)+\epsilon R \pi \tilde{k}_{x}\left(\delta \rho_{k_{x}, k_{y}+1}-\delta \rho_{k_{x}, k_{y}-1}\right) \\
\frac{\partial \delta u_{k_{x}, k_{y}}(t)}{\partial t}= & -\pi \epsilon R\left(\delta v_{k_{x}, k_{y}+1}+\delta v_{k_{x}, k_{y}-1}\right)+\pi \epsilon R \tilde{k}_{x}\left(\delta u_{k_{x}, k_{y}+1}-\delta u_{k_{x}, k_{y}-1}\right) \\
& -4 \pi^{2} \epsilon\left(\tilde{k}_{x}^{2}+k_{y}^{2}\right) \delta u_{k_{x}, k_{y}}-4 \pi^{2} \alpha \epsilon \tilde{k}_{x}\left(\tilde{k}_{x} \delta u_{k_{x}, k_{y}}+k_{y} \delta v_{k_{x}, k_{y}}\right) \\
& +2 \pi i \tilde{k}_{x} \delta \rho_{k_{x}, k_{y}}+F_{k_{x}, k_{y}}(t)
\end{aligned}
$$




$$
\begin{aligned}
\frac{\partial \delta v_{k_{x}, k_{y}}(t)}{\partial t}= & \pi \epsilon R \tilde{k}_{x}\left(\delta v_{k_{x}, k_{y}+1}-\delta v_{k_{x}, k_{y}-1}\right)-4 \pi^{2} \epsilon\left(\tilde{k}_{x}^{2}+k_{y}^{2}\right) \delta v_{k_{x}, k_{y}} \\
& -4 \pi^{2} \alpha \epsilon k_{y}\left(\tilde{k}_{x} \delta u_{k_{x}, k_{y}}+k_{y} \delta v_{k_{x}, k_{y}}\right)+2 \pi i k_{y} \delta \rho_{k_{x}, k_{y}}+G_{k_{x}, k_{y}}(t) .
\end{aligned}
$$

where $\alpha=\zeta / \eta$ and

$$
\epsilon=\frac{\nu}{c_{s} L_{y}}
$$

The functions $F_{k_{x}, k_{y}}$ and $G_{k_{x}, k_{y}}$ are Fourier components of the noise terms ; their covariances follow directly from eqs. (20, 21):

$$
\begin{aligned}
& <F_{k_{x}, k_{y}}(t) F_{k_{x}^{\prime}, k_{y}^{\prime}}\left(t^{\prime}\right)>=\epsilon A\left[(\alpha+1) \tilde{k}_{x}^{2}+k_{y}^{2}\right] \delta_{\mathbf{k}+\mathbf{k}^{\prime}, 0}^{K r} \delta\left(t-t^{\prime}\right), \\
& <F_{k_{x}, k_{y}}(t) G_{k_{x}^{\prime}, k_{y}^{\prime}}\left(t^{\prime}\right)>=\epsilon A \alpha \tilde{k}_{x} k_{y} \delta_{\mathbf{k}+\mathbf{k}^{\prime}, 0}^{K r} \delta\left(t-t^{\prime}\right), \\
& <G_{k_{x}, k_{y}}(t) G_{k_{x}^{\prime}, k_{y}^{\prime}}\left(t^{\prime}\right)>=\epsilon A\left[\tilde{k}_{x}^{2}+(\alpha+1) k_{y}^{2}\right] \delta_{\mathbf{k}+\mathbf{k}^{\prime}, 0}^{K r} \delta\left(t-t^{\prime}\right),
\end{aligned}
$$

where $\mathbf{k} \equiv\left(k_{x} / a_{r}, k_{y}\right)$ and

$$
A=8 \pi^{2} \frac{k_{B} T_{0}}{M c_{s}^{2}},
$$

$M=a_{r} \rho_{0} L_{y}{ }^{2}$ being the total mass of the system.

The analysis of the above Langevin equations can be simplified somewhat by noticing that the quantity $\epsilon$ must remain small if one wishes to remain within the limit of validity of the hydrodynamic regime [25]. Furthermore, as already mentioned in the introduction, in this article we limit ourselves to strictly sub-sonic flows, so that $\epsilon R=u_{0} / c_{s}<<1$. We thus have at our disposal a natural small parameter which, however, have to be used with care since the solution of the Langevin equations (23- 25) proves to be singular in the limit $\epsilon \rightarrow 0$. Moreover, it turns out that the behavior of the system is qualitatively independent of the value of the bulk viscosity coefficient so that, to avoid cumbersome notations, we simply set $\alpha=0$ (recall that $\alpha=\zeta / \eta$ ). In any case, the calculations remain lengthy and tedious, so that here we concentrate mainly on the final results, giving only a brief sketch of the intermediate steps. 
We pay particular attention to two quantities. First, the so-called scattering function, defined as the space-time Fourier transform of the density auto-correlation function:

$$
\begin{gathered}
S_{\mathbf{k}}(\omega)=\int_{-\infty}^{+\infty} d t \exp (i \omega t)<\delta \rho_{\mathbf{k}}(t) \delta \rho_{-\mathbf{k}}(0)> \\
<\delta \rho_{\mathbf{k}}(t) \delta \rho_{-\mathbf{k}}(0)>=\frac{1}{S^{2}} \iint d \mathbf{r} d \mathbf{r}^{\prime} \exp \left\{2 \pi i \mathbf{k} \cdot\left(\mathbf{r}-\mathbf{r}^{\prime}\right)\right\}<\delta \rho(\mathbf{r}, t) \delta \rho\left(\mathbf{r}^{\prime}, 0\right)>
\end{gathered}
$$

where the integrals extend over the surface $S=a_{r} \times 1$ of the system. Next, the space-time Fourier transform of the velocity auto-correlation function, defined in a similar fashion:

$$
W_{\mathbf{k}}(\omega)=\int_{-\infty}^{+\infty} d t \exp (i \omega t)<\delta \mathbf{v}_{\mathbf{k}}(t) \cdot \delta \mathbf{v}_{-\mathbf{k}}(0)>
$$

as well as its static (equal time) counterpart:

$$
<\delta \mathbf{v}_{\mathbf{k}} \cdot \delta \mathbf{v}_{-\mathbf{k}}>=\frac{1}{2 \pi} \int_{-\infty}^{+\infty} d \omega W_{\mathbf{k}}(\omega)
$$

We found that, to dominant order in $\epsilon, S_{\mathbf{k}}(\omega)$ is not affected by the nonequilibrium constraints, i.e.

$$
S_{\mathbf{k}}(\omega)=\frac{32 \epsilon A k^{4} \pi^{4}}{\left(\omega^{2}-4 k^{2} \pi^{2}\right)^{2}+16 \epsilon^{2} \omega^{2} k^{4} \pi^{4}}\left[1+O\left(\epsilon^{2} R^{2}\right)\right]
$$

where $k^{2} \equiv\left(\tilde{k}_{x}^{2}+k_{y}^{2}\right)$. We note that the scattering function exhibits only sound mode peaks (Brillouin lines). The absence of a purely dissipative mode around $\omega \approx 0$ (Rayleigh line) is directly related to the fact that the Kolmogorov flow is "strictly" isothermal, i.e. there are no temperature (or entropy) fluctuations. On the other hand, the velocity auto-correlation function do exhibit a purely dissipative viscous regime around $\omega \approx 0$, together with a sound regime located around $\omega \approx \pm 2 \pi|k|$. Here again we found that, to dominant order in $\epsilon$, the sound regime is not affected by the nonequilibrium constraints and behaves very much like the scattering function, eq. (33). One thus arrives at the conclusion that the nonequilibrium constraints affect mainly the behavior of the fluid near the origin $\omega \approx 0$ (the viscous regime).

We first consider near equilibrium situations, limiting ourselves to relatively small values of the Reynolds number $R$. In this case the Langevin eqs. 23 - 25) can be solved perturbatively by expanding the hydrodynamic variables around the equilibrium, 


$$
\begin{gathered}
\delta \rho=\delta \rho_{e q}+\mu \delta \rho_{1}+\mu^{2} \delta \rho_{2}+\ldots \\
\delta \mathbf{v}=\delta \mathbf{v}_{e q}+\mu \delta \mathbf{v}_{1}+\mu^{2} \delta \mathbf{v}_{2}+\ldots
\end{gathered}
$$

where the subscript "eq" denotes equilibrium quantities and the parameter $\mu$ is defined as $\mu \equiv R / 2 \pi$. After some tedious algebra, one gets for the static correlation function:

$$
\begin{gathered}
<\delta \mathbf{v}_{\mathbf{k}} \cdot \delta \mathbf{v}_{-\mathbf{k}}>-<\delta \mathbf{v}_{\mathbf{k}} \cdot \delta \mathbf{v}_{-\mathbf{k}}>_{e q}= \\
(R / 2 \pi)^{2} \frac{A\left(10+2 \tilde{k}_{x}^{6}+5 \tilde{k}_{x}^{4}+\tilde{k}_{x}^{2}\right)}{2\left(\tilde{k}_{x}^{2}+4\right)\left(2 \tilde{k}_{x}^{2}+5\right)\left(2 \tilde{k}_{x}^{2}+1\right)\left(\tilde{k}_{x}^{2}+1\right)^{2}}\left[1+O\left(\epsilon^{2},(R / 2 \pi)^{2}\right)\right]
\end{gathered}
$$

where $\left\langle\delta \mathbf{v}_{\mathbf{k}} \cdot \delta \mathbf{v}_{-\mathbf{k}}\right\rangle_{e q}=2 A$ is the equilibrium contribution and, to simplify the presentation, we have considered the case $k_{y}=1$.

To check the validity of this result, we have solved numerically the Langevin eqs. (23-25). The traditional procedure consists of simulating the corresponding stochastic processes and using the hydrodynamical sample paths (time series) so obtained to construct the various correlation functions. Unavoidable for nonlinear problems, this procedure is quite simple to set up but requires very long runs in order to get reliable statistics. Alternatively, one can solve directly the equations governing the evolution of the correlation functions which can be obtained easily from the underlying Langevin equations [26]. This latter procedure is accurate (no need for statistics) and quite fast but it is, of course, limited to linear problems. We have used both techniques, the former to simulate the full nonlinear hydrodynamic equations (3, 4) with noisy stress tensor, and the latter to study the statistical properties of the (linear) Langevin equations (23 - 25), limiting ourselves to the first $41 k_{y}$ modes (that is $\delta \rho_{k_{x}, k_{y}}(t)=\delta \mathbf{v}_{k_{x}, k_{y}}(t) \approx 0$ for $\left.\left|k_{y}\right| \geq 21\right)$.

In figure 1 we have presented the static velocity auto-correlation function, as given by the relation (35), together with the corresponding numerical solution. As it can be seen, quantitative agreement is demonstrated for $R \leq 4$ but discrepancies gradually appear as we consider larger values of the Reynolds number. This is to be expected since the validity of the relation (35) can only be guaranteed for "small" values of the Reynolds number. 
Before discussing the behavior of the system for larger values of $R\left(<R_{c}\right)$ it is instructive to study the properties of the static correlation function in real space, $\langle\delta \mathbf{v}(\mathbf{r}) \cdot \delta \mathbf{v}(0)>$. This can be obtained by summing the product $\left\langle\delta \mathbf{v}_{\mathbf{k}} \cdot \delta \mathbf{v}_{-\mathbf{k}}>\times \exp \left[2 \pi i\left(x \tilde{k}_{x}+y k_{y}\right)\right]\right.$ over $\left(k_{x}, k_{y}\right)$. Analytical calculations, however, prove to be extremely difficult to handle for the general case. We therefore limit ourselves to a special case where only one of the wavenumber is summed over, the other being held fixed. Specifically, we simply set $k_{y}=0$ to obtain:

$$
<\delta \mathbf{v}_{k_{x}} \cdot \delta \mathbf{v}_{-k_{x}}>-2 A=(R / 2 \pi)^{2} \frac{A / 2}{\left(\tilde{k}_{x}^{2}+1\right)\left(1+2 \tilde{k}_{x}^{2}\right)}\left[1+O\left(\epsilon^{2},(R / 2 \pi)^{2}\right)\right]
$$

Note that setting $k_{y}=0$ is equivalent to taking the spatial average over the $y$ direction (cfr. eq. (30)), so that relation (36) holds only for $k_{x} \neq 0$. In fact, $\delta \mathbf{v}_{0,0}(t) \equiv 0$ since the linear momentum of the center of mass is a conserved quantity. With this restriction in mind, the summation can be performed in a straightforward manner to give

$$
\begin{gathered}
<\delta \mathbf{v}(x) \cdot \delta \mathbf{v}(0)>-2 A\left[\delta(x)-a_{r}\right]= \\
\frac{A R^{2} a_{r}}{8 \pi}\left[\sqrt{2} \frac{\cosh \left[\sqrt{2} \pi\left(x-a_{r} / 2\right)\right]}{\sinh \left(\pi a_{r} / \sqrt{2}\right)}-\frac{\cosh \left[2 \pi\left(x-a_{r} / 2\right)\right]}{\sinh \left(\pi a_{r}\right)}-\frac{1}{\pi a_{r}}\right]
\end{gathered}
$$

where the second term on the left hand side is the equilibrium contribution [27]. Note the presence of a constant term in both equilibrium and nonequilibrium (right hand side) part which ensures the conservation of the linear momentum.

The nonequilibrium contribution to $\langle\delta \mathbf{v}(x) \cdot \delta \mathbf{v}(0)>$ exhibits long-range correlations since the correlation length is clearly of the order of system's size. This is shown in figure (2) for $R=3$ where quantitative agreement with numerical results is observed. The existence of long-range correlations is generic for fluids under shear constraints and have been predicted by several authors [3, 27], and confirmed by both microscopic [11] and lattice-gas automata simulations [28]. On the other hand, experimental evidence has been so far reported only for fluids under a temperature gradient, where quantitative agreement with fluctuating hydrodynamics was demonstrated [8].

Let us now consider far from equilibrium situations. As pointed out in the last section, for $R$ close to $R_{c}$ one can reasonably well rely on the "3-modes" approximation theory (that 
is $\delta \rho_{k_{x}, k_{y}}(t)=\delta \mathbf{v}_{k_{x}, k_{y}}(t) \approx 0$ for $\left|k_{y}\right| \geq 2$ ). As a consequence, equations (23- 25) reduce to a system of nine coupled linear Langevin equations for each fixed $k_{x}$ which, for consistency,

must be limited to $\left|k_{x}\right| \leq a_{r}$. The calculations can nevertheless be done, leading to the following expression for the static velocity auto-correlation function:

$$
<\delta \mathbf{v}_{\mathbf{k}} \cdot \delta \mathbf{v}_{-\mathbf{k}}>-2 A=\frac{A R^{2}}{2\left(R_{c}^{2}-R^{2}\right)\left(1+2 \tilde{k}_{x}^{2}\right)}\left[1+O\left(\epsilon^{2} R^{2}\right)\right]
$$

where the second term on the left hand side is the equilibrium contribution, $R_{c}\left(k_{x}\right)$ is given by eq. (17) and $k_{y}=1$. The nonequilibrium part diverges as $R \rightarrow R_{c}\left(k_{x}=a_{r}\right)$, but then, of course, the linearized Langevin equations ceases to be valid. In figure 3 we have represented the result (38) for increasing values of $R$, together with the numerical solution of the linear Langevin equations (23) - 25) as well as the results obtained by simulation of the full nonlinear hydrodynamic equations (3, (4) with noisy stress tensor. Quantitative agreement is observed for values of $R$ up to 12 , but significant discrepancies start to show up as $R \rightarrow R_{c}(\approx 12.87)$ where the linearized theory leads to diverging correlation function (cfr. eq. (38)). This is not the case for the correlation function based on the full nonlinear equations which seems to exhibit a maximum around $R_{c}$. It should however be noted that, due to slowing down of the relaxation of the "critical" Fourier modes, statistical errors are quite important for $R$ close to $R_{c}$ (about $15 \%$ for the last four data), so that no definitive conclusion can be drawn at this stage. In any case, the analysis of the statistical properties of the nonlinear regime is beyond the scope of the present work.

\section{VALIDITY OF THE INCOMPRESSIBILITY ASSUMPTION}

The macroscopic studies of sub-sonic hydrodynamical instabilities are based on the incompressibility assumption which is fundamentally inconsistent with the very foundation of the fluctuating hydrodynamics formalism [13]. For instance, it is easy to show that at equilibrium $(R=0)$, one has:

$$
\frac{U_{\mathbf{k}}(\omega)_{e q}}{V_{\mathbf{k}}(\omega)_{e q}}=\frac{\left(\omega^{2}-4 \pi^{2} k_{y}^{2}\right)^{2}+16 \pi^{4} \tilde{k}_{x}^{2} k_{y}^{2}}{\left(\omega^{2}-4 \pi^{2} \tilde{k}_{x}^{2}\right)^{2}+16 \pi^{4} \tilde{k}_{x}^{2} k_{y}^{2}}\left[1+O\left(\epsilon^{2}\right)\right]
$$


where $U_{\mathbf{k}}(\omega)$ and $V_{\mathbf{k}}(\omega)$ are the space-time Fourier transforms of $<\delta u(\mathbf{r}, t) \delta u\left(\mathbf{r}^{\prime}, 0\right)>$ and $<\delta v(\mathbf{r}, t) \delta v\left(\mathbf{r}^{\prime}, 0\right)>$ respectively. On the other hand, the incompressibility assumption, eq. (घ), implies

$$
U_{\mathbf{k}}(\omega)_{i n c} / V_{\mathbf{k}}(\omega)_{i n c}=k_{y}^{2} / \tilde{k}_{x}^{2}
$$

where the subscript "inc" refers to incompressible fluids. Except near the origin $\omega \approx 0$ (long time limit), this result is clearly in contradiction with the correct equilibrium form, eq. (39). In particular, the equilibrium static auto-correlations are independent of the wave vector,

$$
<\delta u_{\mathbf{k}} \delta u_{-\mathbf{k}}>_{e q}=<\delta v_{\mathbf{k}} \delta v_{-\mathbf{k}}>_{e q}=A
$$

whereas relation (40) leads to $<\delta u_{\mathbf{k}} \delta u_{-\mathbf{k}}>_{\text {inc }} /<\delta v_{\mathbf{k}} \delta v_{-\mathbf{k}}>_{i n c}=k_{y}^{2} / \tilde{k}_{x}^{2}$.

The situation is somewhat different for the nonequilibrium case. As we have shown in the previous section, to dominant order in $\epsilon$ both the scattering function and the sound regime of the velocity auto-correlation function assume their equilibrium form, regardless of the value of the Reynolds number. The nonequilibrium constraints thus affect mainly the behavior of the fluid near the origin $\omega \approx 0$ (the viscous regime). This result has an interesting consequence. It suggests that, as far as the nonequilibrium properties of the fluid are concerned, one may rely on the incompressibility assumption, eq. (7), since the compressibility of the fluid affects mainly the sound modes which are well separated from the purely viscous modes (recall that $\epsilon$ is small). Analytical calculations confirm the above arguments and lead to the following relation:

$$
\frac{U_{\mathbf{k}}(\omega)-U_{\mathbf{k}}(\omega)_{e q}}{V_{\mathbf{k}}(\omega)-V_{\mathbf{k}}(\omega)_{e q}}=\frac{k_{y}^{2}}{\tilde{k}_{x}^{2}}\left[1+O\left(\epsilon^{2} R^{2}\right)\right]
$$

where both the numerator and the denominator in the left hand side prove to assume a Lorentzian shape, sharply peaked around the origin (the width is of the order of $\epsilon$ ), since the sound regime cancels out. Nevertheless, because of the presence of the equilibrium contributions, the relation (42) is still in contradiction with the incompressibility condition, eq. (40). There exist, however, two different situations where this objection can be ruled out. 
First, near the origin $(\omega \approx 0)$ where the fluid satisfies the incompressibility condition already at equilibrium, i.e. $U_{\mathbf{k}}(\omega)_{e q} \tilde{k}_{x}^{2} \approx V_{\mathbf{k}}(\omega)_{e q} k_{y}^{2}$. Obviously, this situation concerns only the long time behavior of the fluid. For instance, the static correlation functions obey

$$
\frac{<\delta u_{\mathbf{k}} \delta u_{-\mathbf{k}}>-A}{<\delta v_{\mathbf{k}} \delta v_{-\mathbf{k}}>-A}=\frac{k_{y}^{2}}{\tilde{k}_{x}^{2}}\left[1+O\left(\epsilon^{2} R^{2}\right)\right]
$$

which contradicts the incompressibility condition, eq. (40).

A more interesting situation concerns the behavior of the fluid near the instability where it can be shown that in the limit $R \rightarrow R_{c}$ both the static and dynamic velocity correlation functions behave as $O\left(\left(R_{c}^{2}-R^{2}\right)^{-1}\right)$. In other words, for $R$ "close" enough to $R_{c}$, equilibrium contributions become negligible so that the fluid behaves basically in an incompressible way.

It should however be realized that this appealing conclusion is based on the linearized Langevin equations (23-25) which are not valid near the convective instability. The study of the statistical properties of the system in the critical regime requires a nonlinear analysis of the fluctuating equations which is beyond the scope of the present work and will be reported elsewhere. Instead, we resort here to numerical analysis only. More precisely, we have simulated the full nonlinear fluctuating hydrodynamic equations to obtain the ratio of the $x$ and $y$ components of the static velocity auto-correlation function for several values of the Reynolds number. The results are depicted in figure 4 for $k_{x}=k_{y}=1, a_{r}=2$, so that the expected value of this ratio for an incompressible fluid is 4 . This is precisely what we observe, but only for values of $R \geq 12.8$ (recall that $R_{c} \approx 12.87$ ), a domain which is beyond the validity of linearized hydrodynamic equations (cfr. previous section).

\section{CONCLUDING REMARKS}

Our main purpose in this article was the study of the statistical properties of the linearized Kolmogorov flow. The simplicity of this model allows a detailed analysis of the fluctuations spectrum from near equilibrium up to the vicinity of the first instability leading to convective rolls. For this latter case, the analytical calculations were based on a 
"3-modes" approximation theory, which consists in retaining only the Fourier modes with wavenumber $\left|k_{y}\right| \leq 1$, while for the former case we have set up a perturbation scheme around the equilibrium. Extensive numerical calculations allow us to emphasize clearly the limit of validity of both regimes. In particular, we have shown that the "3-modes" approximation theory holds already for $R \geq 8$ and leads to a diverging velocity auto-correlation function as $R \rightarrow R_{c}$. On the other hand, the simulation of the full nonlinear fluctuating hydrodynamic equations indicates that the validity of linearized hydrodynamic equations can be guaranteed for Reynolds number as high as $12\left(R_{c} \approx 12.87\right)$.

Another interesting result concerns the validity of the incompressibility assumption which greatly simplifies the mathematical analysis of the problem. The compressibility of a fluid affects mostly fast sound modes whereas the dynamic of the system near an instability is expected to be governed mainly by dissipative slow modes. This intuitive argument has been used by many authors who have considered fluctuating incompressible hydrodynamic equations, or even directly the corresponding normal form amplitude equations to which they added random noise terms [29]. In these approaches, the characteristics of the noise terms cannot be related to equilibrium statistical properties of the fluid and thus remain arbitrary. A more satisfactory approach would be to start with the compressible fluctuating hydrodynamic equations. Such a procedure, however, proves to be extremely difficult mainly because of the boundary value problem. To our knowledge, the only attempt in this direction has been made by Schmitz and Cohen for the case of Bénard instability «14. Concentrating on the behavior of a small layer in the bulk, these authors have succeeded in deriving the linearized fluctuating equations close to the convective instability.

Here again, the relative simplicity of the Kolmogorov flow allows some further progress in this important issue. In this respect, we have shown that in the long time limit the flow behaves as an incompressible fluid, regardless of the value of the Reynolds number. This, however, proves to be not the case for the short time behavior. In particular, the incompressibility assumption leads in general to a wrong form of the static correlation functions. The only exception is near the convective instability, where we have shown that the 
incompressibility assumption remains valid.

The problem with this conclusion is that the linearized fluctuating hydrodynamic equations, on which this whole paper is based, are no more valid close to the instability threshold. Although extensive numerical simulations have basically confirmed our predictions, a full answer to this important problem requires nevertheless a nonlinear analysis of the fluctuating Kolmogorov flow. Work on this direction is in progress.

\section{ACKNOWLEDGMENTS}

We are very grateful to professors E. G. D. Cohen, G. Nicolis, J. W. Turner and C. Van den Broeck for helpful comments. One of us (I. B.) acknowledges successive support from the EC TEMPUS program, the Center for Nonlinear Phenomena and Complex Systems of the Free University of Brussels, the GCIR of the French Community in Belgium, and the IUAP, Prime Minister's Office of the Belgian Government. 


\section{REFERENCES}

[1] L. D. Landau and E. M. Lifshitz, "Fluid Mechanics", Pergamon Press, Oxford (1984).

[2] T. R. Kirkpatrick, E. G. D. Cohen and J. R. Dorfman, Phys. Rev. Lett., 42, 862 (1979) ; ibid. 44, 472 (1980) ; ibid. Phys. Rev. A 26, 995 (1982).

[3] J. Dufty, in Spectral Line Shapes, P. Wende ed., De Kruger, Berlin (1981) ; J. Lutsko and J. Dufty, Phys.Rev. A 32, 1229 (1985).

[4] I. Procaccia, D. Ronis, I. Oppenheim, Phys. Rev. Lett.42, 287 (1979) ; D. Ronis, I. Procaccia and I. Oppenheim, Phys. Rev. A 19, 1324 (1979) ; A. -M. Tremblay, M. Arai and E. Siggia, Phys. Rev. A 23, 1451 (1981) ; D. Ronis, I. Procaccia and I. Oppenheim, Phys. Rev. A 26, 1812 (1982).

[5] G. Van der Zwan, D. Bedeaux and P. Mazur, Physica A, 107, 491 (1981) ; R. Schmitz, and E.G.D. Cohen, J.Stat.Phys. 39, 285 (1985) ; ibid. 40, 431 (1985).

[6] J. Machta, I. Procaccia and I. Oppenheim, Phys. Rev. Lett., 42, 1368 (1979) ; J. Machta, I. Oppenheim and I. Procaccia, Phys. Rev. A., 22, 2809 (1980).

[7] R. Schmitz, Phys. Reports, 171, 1 (1988).

[8] B.M. Law, and J.V. Sengers, J.Stat.Phys. 57, 531 (1989);

B.M. Law, P.N. Segré, R.W. Gammon, and J.V. Sengers, Phys.Rev. A 41, 816 (1990).

[9] M. Malek Mansour, A.L. Garcia, G.Lie, and E. Clementi, Phys.Rev.Lett., 58, 874 (1987) ; M. Mareschal, M. Malek Mansour, G. Sonino, and E. Kestemont, Phys.Rev. A 45, 7180 (1992).

[10] A. Suárez, J. P. Boon and P. Grosfils, Phys. Rev. E 54, 1208 (1996).

[11] A.L. Garcia, M. Malek Mansour, G. Lie, M. Mareschal, and E. Clementi, Phys.Rev. A 36, 4348 (1987). 
[12] S. Chandrasekhar, "Hydrodynamic and Hydromagnetic Stability", Clarendon Press, Oxford (1961).

[13] V.M. Zaitsev, and M.I. Shliomis, Sov.Phys. JETP 32, 866 (1971).

[14] R. Schmitz, and E.G.D. Cohen, J. Stat. Phys., 46, 319 (1987) ; ibid. Phys. Rev. A 35, $2602(1987)$.

[15] H. Van Beijeren and E. D. G. Cohen, Phys. Rev. Lett. 60, 1208 (1988) ; ibid. J. Stat. Phys. 53, 77 (1988).

[16] A.M. Obukhov, Russ. Math. Survey 38,113 (1983).

[17] S. Gama, M. Vergassola, and U. Frisch, J.Fluid Mech. 260, 95 (1994).

[18] E. N. Lorenz, J. Fluid Mech., 55, 545 (1972).

[19] Z.S. She, Phys. Lett. A 124, 161 (1987); ibid. Ph.D. Thesis, Univ. Paris VII (1987) ; G.I. Sivashinsky, Physica D 17, 243 (1985).

[20] R. Benzi, S. Succi, J. Stat. Phys. 56, 69 (1989) ; U. Frisch, B. Legras, B. Villone, Physica D 94, 36 (1996).

[21] L. Meshalkin, and Ya.G. Sinai, J.Appl.Math.Mech.(P.M.M.) 25, 1140 (1961).

[22] J.S.A. Green, J.Fluid Mech. 62, 273 (1974).

[23] This result is not new and have been obtained by several authors, like for instance A.A. Nepomniaschichii, J. Appl. Math. Mech. 40, 836 (1976), Meshalkin and Sinai [21], and Lorenz [18].

[24] C. Marchioro, Commun.Math.Phys 105, 99 (1986).

[25] See for instance W. E. Alley and B. J. Alder, Phys. Rev. A 27, 3158 (1983) ; W. E. Alley, B. J. Alder and S. Yip, Phys. Rev. A 27, 3174 (1983).

[26] A. L. Garcia, M. Malek Mansour, G. Lie, and E. Clementi, J.Stat.Phys. 47, 209 (1987). 
[27] M. Malek Mansour, J.W. Turner, A.L. Garcia, J.Stat.Phys. 48, 1157 (1987).

[28] J.P.Boon, and D. Hanon (pre-print).

[29] R. Graham, Phys. Rev. A 10, 1762 (1974) ; R. Graham and H. Pleiner, Phys. Fluids, 18, 130 (1975); J. Swift and P. C. Hohenberg, Phys. Rev. A 15, 319 (1977). 


\section{FIGURES}

FIG. 1. Fourier transform of the nonequilibrium part of the static velocity auto-correlation function, normalized by the corresponding equilibrium part, as a function of the Reynolds number. The solid curve represents the theoretical prediction, as given by eq. (35), whereas the diamonds correspond to results obtained by numerical simulation of the linear Langevin equations (23 - 25). The parameters are : $\epsilon=10^{-2}, A=10^{-3}$ (defined by eq. (28)), $k_{y}=1, k_{x}=1$ (i.e. $\tilde{k}_{x}=1 / 2$ ). The estimated statistical errors are less than $4 \%$.

FIG. 2. Nonequilibrium part of the static velocity auto-correlation function, normalized by the parameter $A$ (defined by eq. (28)), as a function of the spatial coordinate $x$. The solid curve represents the theoretical prediction, as given by eq. (37), whereas the diamonds correspond to results obtained by numerical simulation of the linear Langevin equations (23 - 25). The Reynolds number is set to $R=3$ and the other parameters are as given in the caption of figure (1).

FIG. 3. Fourier transform of the nonequilibrium part of the static velocity auto-correlation function, normalized by the corresponding equilibrium part, as a function of the Reynolds number. The solid curve represents the prediction based on the "3-modes" approximation theory, eq. (38), whereas crosses and diamonds correspond to numerical results obtained respectively by the simulation of linear and nonlinear Langevin equations, eqs. $(3,4)$. The parameters are as given in the caption of figure (1). The estimated statistical errors are about $15 \%$ for the last four data points.

FIG. 4. Ratio of $x$ and $y$ component of the velocity auto-correlation function in Fourier space,

as a function of the Reynolds number. The parameters are : $\epsilon=10^{-2}, A=10^{-6}$ (defined by eq. $(28)), k_{y}=1, k_{x}=1$ (i.e. $\tilde{k}_{x}=1 / 2$ ). The dashed line represents the expected ratio for an incompressible fluid, whereas the diamonds correspond to results obtained by the simulation of the full nonlinear Langevin equations, eq. $(3,4)$. The estimated statistical errors do not exceed $8 \%$. 


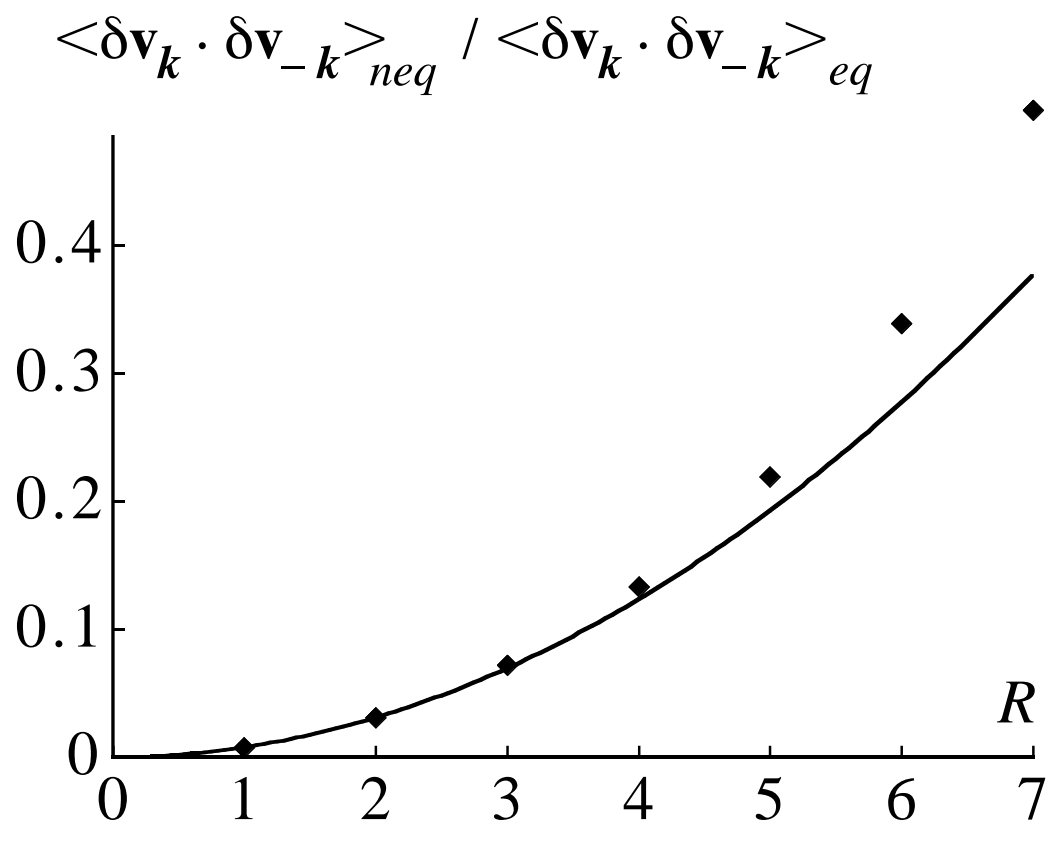




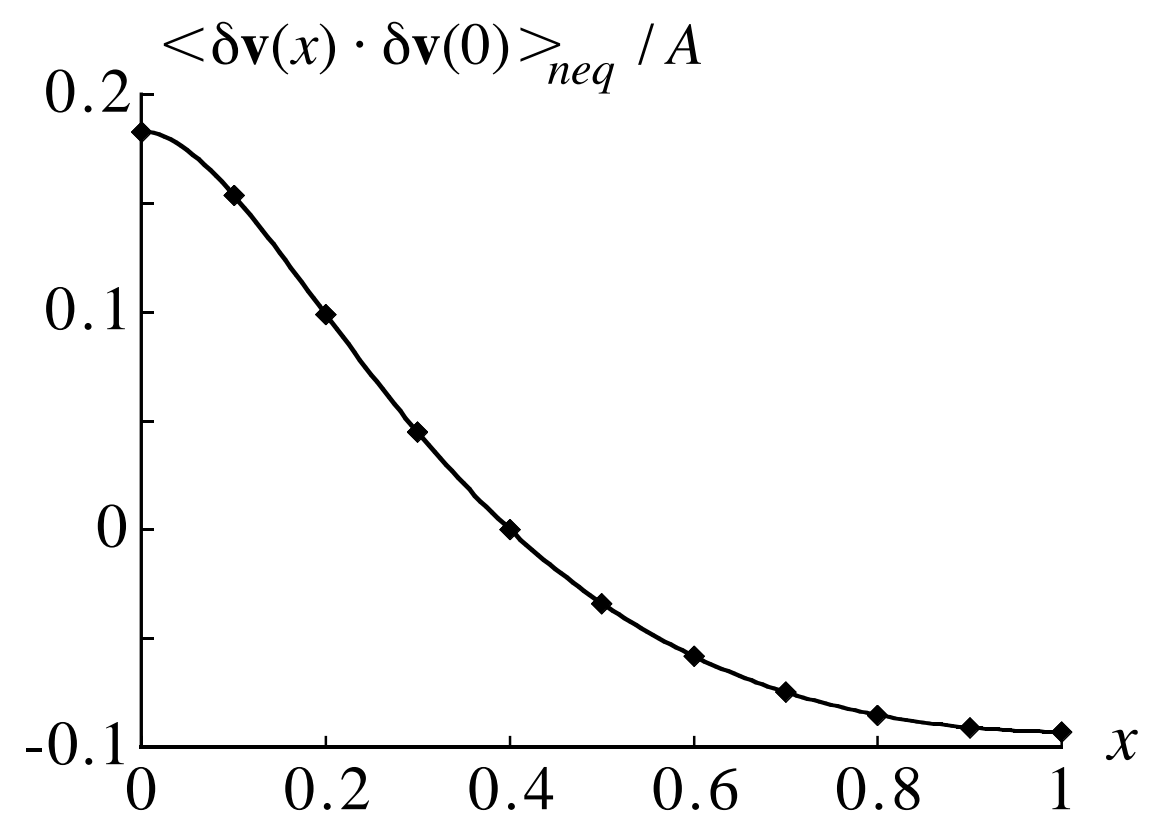




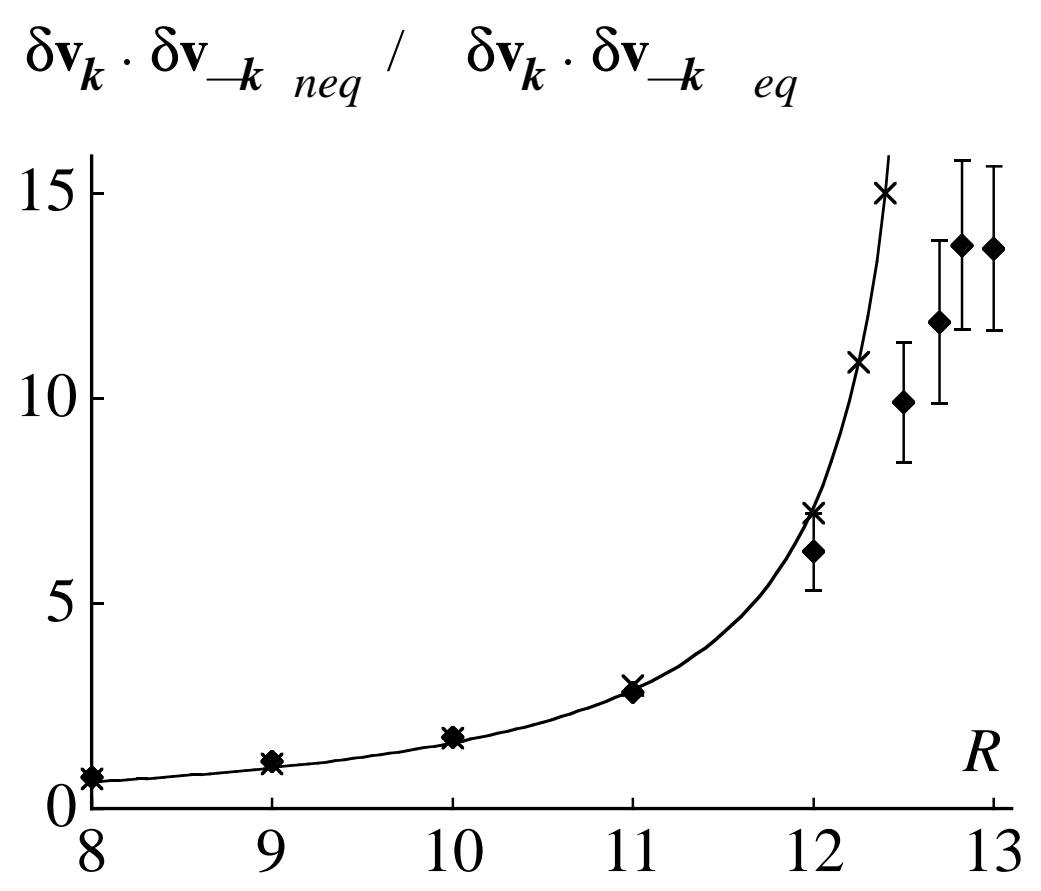




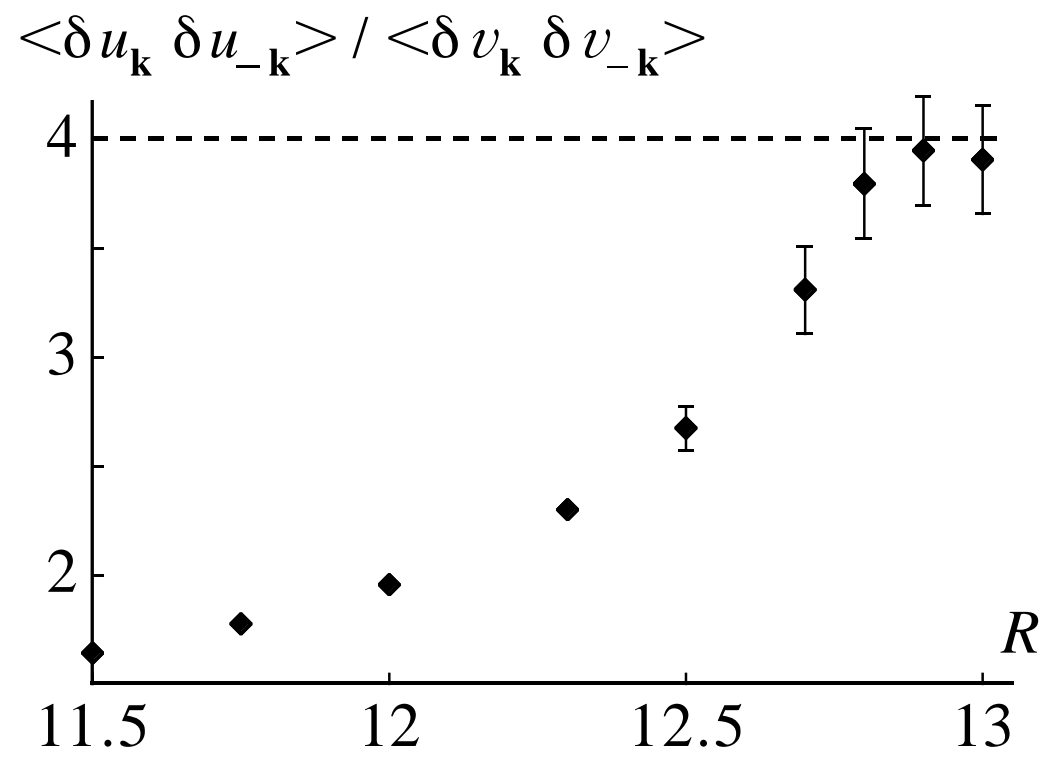

